\title{
Incremental Reverse Nearest Neighbor Ranking in Vector Spaces
}

\author{
Tobias Emrich, Hans-Peter Kriegel, Peer Kröger, Matthias Renz, and Andreas Züfle \\ Institute for Informatics, Ludwig-Maximilians-Universität München \\ Oettingenstr. 67, 80538 München, Germany \\ http://www.dbs.ifi.lmu.de \\ \{emrich,kriegel,kroegerp,renz,zuefle\}@dbs.ifi.lmu.de
}

\begin{abstract}
In this paper, we formalize the novel concept of incremental reverse nearest neighbor ranking and suggest an original solution for this problem. We propose an efficient approach for reporting the results incrementally without the need to restart the search from scratch. Our approach can be applied to a multidimensional feature database which is hierarchically organized by any R-tree like index structure. Our solution does not assume any preprocessing steps which makes it applicable for dynamic environments where updates of the database frequently occur. Experiments show that our approach reports the ranking results with much less page accesses than existing approaches designed for traditional reverse nearest neighbor search applied to the ranking problem.
\end{abstract}

\section{Introduction}

While the reverse nearest neighbor (RNN) search problem, i.e. finding all objects in a database that have a given query $q$ among their corresponding $k$-nearest neighbors, has been studied extensively in the past years, considerably less work has been done so far to support an RNN ranking of objects of a database. An RNN ranking sorts the objects $o$ of the database according to the number of other objects in the database that are more similar to $o$ than $q$. Thus, if an object $o$ has a ranking score of $i$ w.r.t. a query $q$, object $o$ would also be a reverse $k$-nearest neighbor of $q$ for all $k \geq i$ but not a reverse $k$-nearest neighbor of $q$ for all $k<i$.

Initially, the RNN ranking query reports those objects having the smallest ranking scores in a non-deterministic way since several objects may have the same minimal ranking score. Thereby, the results are reported on demand whenever a function called getNext() is invoked. In other words, each consecutive call of getNext() reports one object with minimal ranking score until all objects have been reported.

The major challenge for algorithms that support rankings in general and RNN rankings in particular is that the result of each getNext()-call should be computed incrementally rather than from scratch, i.e. the current state after each getNext()-call needs to be stored and serves as a starting point to compute the results of the next call. The advantage of an incremental ranking method in general is that no parameter $k$ has to be specified for the query in advance and the first (most relevant) results are reported immediately without the overhead of simultaneously computing less relevant results. In 
addition, if the initial results are not sufficient due to any application specific reasons, further results can be requested on demand by calling the getNext() function.

The reminder of this paper is organized as follows. In Section 2 we formally define the RNN ranking problem we want to solve here and discuss related work. Section 3 explores our novel solution to this problem. In Section 4 we present an experimental evaluation. Last but not least, Section 5 concludes the paper.

\section{Survey}

\subsection{Problem Formalization}

In the following, we assume that $\mathcal{D}$ is a database of $n$ feature vectors and dist is the Euclidean distance $^{1}$ on the points in $\mathcal{D}$. In addition, we assume that the points are indexed by any traditional aggregate point access method like the aR-Tree family $[1,2]$. The set of $k$-nearest neighbors of a point $q$ is the smallest set $N N_{k}(q) \subseteq \mathcal{D}$ that contains at least $k$ points such that $\forall o \in N N_{k}(q), \forall \hat{o} \in \mathcal{D}-N N_{k}(q): \operatorname{dist}(q, o)<\operatorname{dist}(q, \hat{o})$. The point $p \in N N_{k}(q)$ with the highest distance to $q$ is called the $k$-nearest neighbor $(k \mathrm{NN})$ of $q$. The distance $\operatorname{dist}(q, p)$ is called $k \mathrm{NN}$ distance of $q$.

The set of reverse k-nearest neighbors $(\mathrm{R} k \mathrm{NN})$ of a point $q$ is then defined as

$$
R N N_{k}(q)=\left\{p \in \mathcal{D} \mid q \in N N_{k}(p)\right\} .
$$

Here, we will be interested in computing a ranking of reverse nearest neighbors (RNNs) w.r.t. a query object $q$ rather than in computing the $\mathrm{R} k \mathrm{NN}$ of $q$ for a fixed value of $k$. Let the function $R: \mathcal{D} \rightarrow \mathbb{N}$ return for an object $o \in \mathcal{D}$ the number of objects which are closer to $o$ than the query $q$, i.e. formally,

$$
R(o)=|\{p \in \mathcal{D}: \operatorname{dist}(p, o)<\operatorname{dist}(q, o)\}| .
$$

Obviously, it holds that $o \in R N N_{k}(q)$ iff $R(o) \leq k$.

The problem of a reverse nearest neighbor ranking is to return incrementally all objects $o \in \mathcal{D}$ in increasing order of the values of $R(o)$ by calling the method getNext(). In case of ties, getNext() may report any qualifying object, i.e. we will allow for nondeterminism. Let us note that the $i$-th call of getNext() not necessarily returns an object that is an $\mathrm{R} i \mathrm{NN}$ of $q$, because for a fixed value of $k$ the set $R N N_{k}(q)-R N N_{k-1}(q)$ generally may contain an (even empty) set of points. In other words, the $i$-th call of getNext() may report an object $o$ with $R(o) \neq i$. As a consequence, as additional information, the result of each of the ranking steps should include not only the actual object $o$ but also its ranking count (ranking score) $R(o)$.

\subsection{Related Work}

The problem of supporting reverse $k$-nearest neighbor $(\mathrm{R} k \mathrm{NN})$ queries efficiently, i.e. computing for a given query $q$ and a number $k$ the R $k$ NNs of $q$, has been studied extensively in the past years. Existing approaches for Euclidean R $k \mathrm{NN}$ search can be

\footnotetext{
${ }^{1}$ The concepts described here can also be extended to any $L_{p}$-norm.
} 


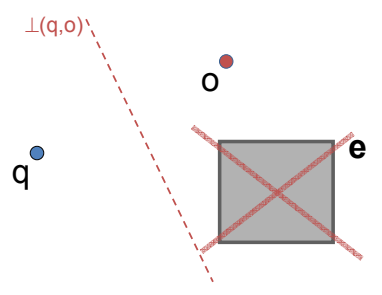

Fig. 1. TPL pruning $(k=1)$.

classified as self-pruning approaches or mutual-pruning approaches. Self-pruning approaches like the RNN-Tree [3] and the RdNN-Tree [4] are usually designed on top of a hierarchically organized tree-like index structure. They try to estimate the $k \mathrm{NN}$ distance of each index entry $e$. If the $k \mathrm{NN}$ distance of $e$ is smaller than the distance of $e$ to the query $q$, then $e$ can be pruned. Thereby, self-pruning approaches do not usually consider other entries (database points or index nodes) in order to estimate the $k \mathrm{NN}$ distance of an entry $e$, but simply precompute $k \mathrm{NN}$ distances of database points and propagate these distances to higher level index nodes. Since the $k \mathrm{NN}$ distances need to be materialized, both approaches are limited to a fixed value of $k$ and cannot be generalized to answer $\mathrm{R} k \mathrm{NN}$-queries with arbitrary values of $k$. In addition, approaches based on precomputed distances can generally not be used when the database is updated frequently. Mutual-pruning approaches such as [5-7] use other points to prune a given index entry $e$. The most general and efficient approach called TPL is presented in [7]. It uses any hierarchical tree-based index structure such as an R-Tree to compute a nearest neighbor ranking of the query point $q$. The key idea is to iteratively construct Voronoi hyper-planes around $q$ w.r.t. to the points from the ranking. Points and index entries that are beyond $k$ Voronoi hyper-planes w.r.t. $q$ can be pruned and need not to be considered for Voronoi construction anymore. The idea of this pruning is illustrated in Figure 1 for $k=1$. Entry $e$ can be pruned, because it is beyond the Voronoi hyper-plane between $q$ and candidate $o$, denoted by $\perp(q, o)$. For the general case, $e$ can be pruned if $e$ is beyond $k$ hyper-planes w.r.t. all current candidates. If $e$ cannot be pruned, it is refined, or, if $e$ is already a database object, $e$ is a new candidate and the hyperplane $\perp(q, e)$ will be considered for pruning in the following. If the ranking queue is empty, the remaining candidate points must be refined, i.e. for each of these candidates, a $k \mathrm{NN}$ query must be launched.

Recently, a method for ranked $\mathrm{R} k \mathrm{NN}$ search has been proposed in [8]. In fact, the authors provide a method for computing the results of an $\mathrm{R} k \mathrm{NN}$ query with fixed $k$ that are ranked according to $k$, i.e. the RiNNs are ranked higher than the $\mathrm{R} j \mathrm{NNs}$ if $i<$ $j \leq k$. This problem is obviously different to the problem of computing an incremental RNN ranking which will be adressed here.

Beside solutions for Euclidean data, solutions for general metric spaces (e.g. [911]) usually implement a self-pruning approach. Typically, metric approaches are less efficient than the approaches tailored for Euclidean data because they cannot make use of the Euclidean geometry. 


\section{Incremental RNN Ranking}

Our approach is based on an index structure $\mathcal{I}$ for point data which is based on the concept of minimal-bounding-rectangles, e.g. the R-tree family like [12-14]. In particular, we use multi-resolution aggregate versions of these indexes as described in $[1,2]$ that e.g. aggregate for each index entry $e$ the number of objects that are stored in the subtree with root $e$. The set of objects managed in the subtree of an index entry $e \in \mathcal{I}$ is denoted by subtree $(e)$. Note that the entry $e$ can be an intermediate node in $\mathcal{I}$ or a point, i.e. an object in $\mathcal{D}$. In the latter case, subtree $(e)=\{e\}$.

The general idea of our solution is based on the TPL-like [7] pruning of entries that are beyond a given number of Voronoi hyperplanes. However, instead of pruning an index entry $e$, we need to estimate the ranking count value $R(o)$ for all points $o \in \operatorname{subtree}(e)$. The key observation is that if an index entry $e$ is beyond a Voronoi hyperplane w.r.t. $q$, then we know that for all $o \in \operatorname{subtree}(e)$, the value of $R(o)$ can be increased by one. For example, in Figure 1, entry $e$ is beyond the Voronoi hyperplane between $q$ and $x$, denoted by $\perp(q, x)$. Thus, $x$ will have a smaller distance to all objects $o \in \operatorname{subtree}(e)$ than $q$, i.e. all objects $o \in \operatorname{subtree}(e)$ will have a ranking count $R(o)$ of at least 1 . Simply speaking, the ranking count $R(o)$ of any object $o \in \mathcal{D}$ equals the number of Voronoi hyperplanes (including $\perp(q, o)$ ) that divide the data space such that $o$ and $q$ are in different half spaces.

In the following, we will extend this idea in several important aspects:

- First, we will extend the concept of Voronoi hyperplanes presented in [7] to higher levels of the index. Originally, the TPL approach considers only Voronoi hyperplanes between the query $q$ and another database object, i.e. at least one leaf entry of the index needs to be fully refined before any Voronoi hyperplane is constructed for pruning. Analogously, this would mean that we can only estimate the ranking count values of objects by means of other objects. This will obviously result in a large overhead of unnecessary page accesses. Rather, we will extend the idea of Voronoi-based pruning/ranking to intermediate entries of the index, i.e. we will also consider Voronoi hyperplanes between the query and intermediate index entries.

- Second, we will also integrate the idea of self-pruning in order to estimate the ranking count of objects within a given subtree.

- Third, we further improve the ranking count estimation by taking also partial hyperplane - entry coverings into account. Hyperplanes where an intermediate index entry $e$ is partially beyond them w.r.t. $q$ can also be used to estimate the ranking count of $e$.

The above estimation strategies give us better estimations of the ranking counts which will be important for the ranking algorithm. Last but not least, we will present a ranking algorithm based on the two previously mentioned ideas to estimate the ranking count that incrementally computes the next object of an RNN ranking on demand without recomputing the entire ranking from scratch.

\subsection{Ranking Count Estimation}

Now we explore strategies for estimating the ranking count based on the hyperplane concept. The basic idea of our approach is to apply the ranking count strategy mentioned 


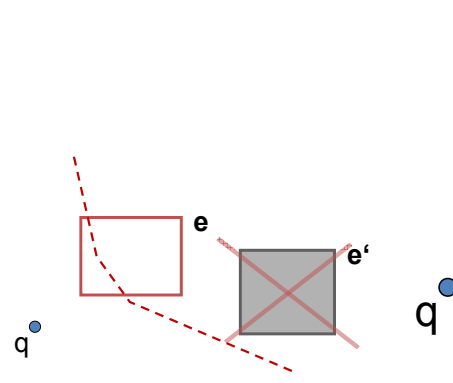

(a) Mutual pruning.

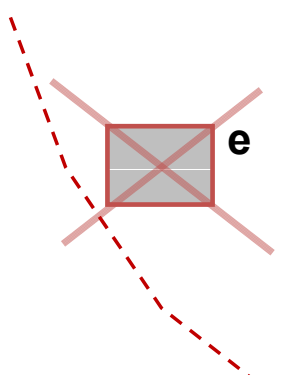

(b) Self pruning.

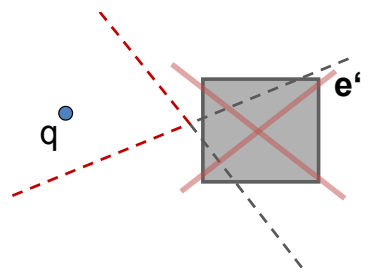

(c) Partial pruning.

Fig. 2. Ranking count estimation based on different pruning strategies.

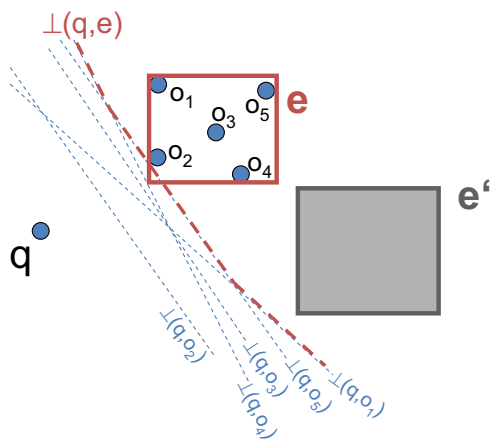

Fig. 3. Conservative approximation $\perp(q, e)$ of the hyperplanes associated with all objects of an index entry $e$.

above during the traversal of the index, i.e. to identify candidates with high ranking counts as early as possible in order to reduce the I/O costs by saving unnecessary page accesses for the computation of the first results. The ability to push candidates to higher ranking positions already at the directory level of the index implies that a directory entry is used to push itself or other entries.

Estimation based on mutual pruning: First, we want to consider the case that a directory entry is used to push other entries back to higher ranking positions by increasing its ranking count. This is similar to the mutual-pruning idea used for R $k$ NN query processing. Generally, the ranking count of an index entry $e \in \mathcal{I}$ can be increased by $k$ according to another entry $e^{\prime} \in \mathcal{I}$ if there are at least $k$ objects in subtree $\left(e^{\prime}\right)$ such that $e$ is behind the Voronoi hyperplane between $q$ and $e^{\prime}$, denoted by $\perp\left(q, e^{\prime}\right)$. In the following a hyperplane associated with an entry/object $e$ is denoted by $\perp(q, e)$.

The key idea of the directory-level-wise ranking count estimation is to identify a hyperplane $\perp(q, e)$ which can be associated with an index entry $e$ and which conservatively approximates the hyperplanes associated with all objects $o_{i}$ in the subtree of $e$, i.e. $o_{i} \in \operatorname{subtree}(e)$. Figure 3 illustrates the idea of this concept. We say that the hyperplane 
associated with an index entry $e$ is related to the set of objects in the subtree of $e$. Since we assume that the number of objects stored in the subtree of an index entry $e$ is known, if we exploit the indexing concept as proposed in [1], we also know for the hyperplane associated with that index entry $e, \perp(q, e)$, how many objects this hyperplane relates to. This means that if an entry/object $e^{\prime}$ is behind a hyperplane $\perp(q, e)$ associated with an index entry $e$, the entry $e^{\prime}$ is also behind all hyperplanes $\perp(q, o)$ associated with the objects $o \in \operatorname{subtree}(e)$. We can use this information in order to increase the ranking count of entries according to $e$ without accessing the child entries of $e$. Consequently, the ranking count of an entry/object $e^{\prime}$ which is behind a hyperplane $\perp(q, e)$ can be increased by $\mid$ subtree $(e) \mid$. In Figure 3 , the ranking count of entry $e^{\prime}$ can be increased by 5 because $\operatorname{subtree}(e)$ contains five points, i.e. $\mid$ subtree $(e) \mid=5$.

Estimation based on self pruning: In addition, we can use these considerations also for increasing the ranking count of an intermediate index entry $e$ by itself. This is similar to the self-pruning idea used for $\mathrm{R} k \mathrm{NN}$ query processing. If an entry $e \in \mathcal{I}$ is behind its own hyperplane, then the ranking count of $e$ can be increased by $\mid$ subtree $(e) \mid-1$, because each object $o \in$ subtree $(e)$ would be behind the hyperplanes associated with all other objects in subtree $(e)$.

Estimation based on partial pruning: The ranking count estimation of an intermediate index entry $e$ can also be based on hyperplanes that do not fully cover $e$. For example, if one part of $e$ is beyond one hyperplane and the other part of $e$ is beyond another hyperplane. In this case, each point in $e$ is at least behind one hyperplane such that the complete entry can be safely moved to a higher ranking position. In general terms, assume that an entry $e \in \mathcal{I}$ is intersected, but not fully covered by $n$ hyperplanes $\perp\left(q, e_{0}\right), \ldots, \perp\left(q, e_{n-1}\right)$ associated with index entries $e_{0} \ldots, e_{n-1}$. Now, the points in $e$ are covered by different numbers of hyperplanes. The ranking count of $e$ can be increased by the minimal number of hyperplanes a point of $e$ is covered by.

Note that the partial pruning based estimation can become very expensive in higherdimensional spaces. The reason is that the determination of the minimal coverage of an index entry w.r.t. all hyperplanes requires complex spatial segmentation operations in order to find the subregions having the same amount of hyperplanes they are behind. This can be very costly in higher-dimensional spaces. In this paper, we propose an efficient approach for the partial pruning based ranking count estimation for the twodimensional space.

In the following, we present solutions for the ranking count estimation according to the above three strategies. First, we show in Section 3.2 how the ranking count estimations can be efficiently computed based on the mutual and self pruning strategies. Next, in Section 3.3, we propose an efficient 2D solution for the partial pruning based estimation.

\subsection{Ranking Count Updates w.r.t. Intermediate Index Entry Hyperplanes}

We first need to determine an entry $e^{\prime} \in \mathcal{I}$ is completely or partially behind a hyperplane $\perp(q, e)$ associated with an entry $e \in \mathcal{I}$. An important observation is that a hyperplane 


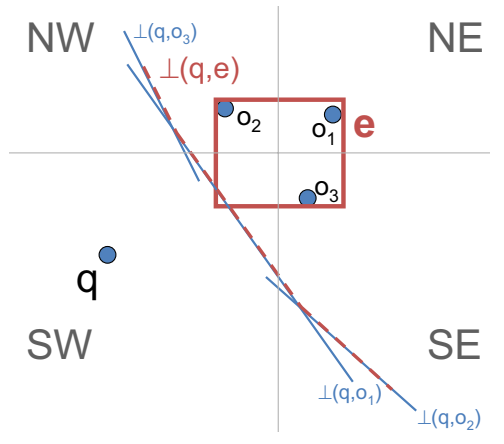

Fig. 4. Computation of conservative hyperplane approximations.

associated with an object $o$ represents all points $p$ which have the same distance to the query point $q$ and to $o$, formally:

$$
\perp(q, o)=\left\{p \in \mathbb{R}^{d}: \operatorname{dist}(p, q)=\operatorname{dist}(p, o)\right\} .
$$

In addition, we know that all objects stored in the subtree of an index entry $e$ are located inside the minimum bounding hyper-rectangle $e . m b r$ that defines the page region of $e$. Thus, we can determine a conservative hyperplane representation of all points stored in the subtree of entry $e$ if we replace the distances between the hyperplane points $p \in \perp(q, e)$ and $o \in$ subtree $(e)$ by the maximum distance between $p$ and the mbr-region of $e$. Consequently, the hyperplanes of all objects $o \in \operatorname{subtree}(e)$ are conservatively approximated by a hyperplane representation consisting of all points in the vector space that fulfill the following condition:

$$
\perp(q, e)=\left\{p \in \mathbb{R}^{d}: \operatorname{dist}(p, q)=\operatorname{MaxDist}(p, e . m b r)\right\} .
$$

In general, a hyperplane representation $H$ is called conservative approximation of a set of hyperplanes $H^{\prime}$, if all objects behind $H$ are definitely behind each hyperplane $h^{\prime} \in H^{\prime}$, formally:

$$
\text { (o behind } H) \Rightarrow\left(\forall h^{\prime} \in H^{\prime}: o \text { behind } h^{\prime}\right)
$$

We can assign such a hyperplane representation to each intermediate entry of our index.

In consideration of the above equations, an index entry $e^{\prime} \in \mathcal{I}$ is defined to be behind a hyperplane $\perp(q, e)$ if the following condition holds:

$$
\forall p \in e . m b r: \operatorname{dist}(p, q)>\operatorname{MaxDist}(p, e . m b r) .
$$

Figure 3 illustrates the conservative approximation $\perp(q, e)$ of all hyperplanes $\perp(q, o)$ for all objects $o \in \operatorname{subtree}(e)$.

In the following we briefly discuss how this conservative approximation $\perp(q, e)$ can be associated with an index entry $e$. An important observation is that a hyperplane associated with an object $o$ represents all points $p$ which have the same distance to the query 


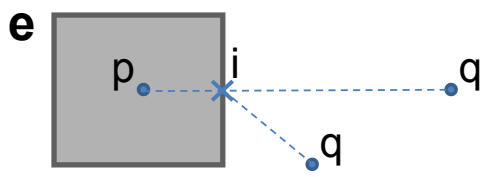

Fig. 5. Example to Lemma 2.

point $q$ and to $o$. In addition, we know that all objects stored in the subtree of an index entry $e$ are located inside the minimum bounding hyper-rectangle (mbr) that defines the page region of $e$. Thus, we can determine a conservative hyperplane representation of all points stored in subtree $(e)$ if we replace the distances between the hyperplane points $p \in \perp(q, e)$ and $o \in \operatorname{subtree}(e)$ by the maximum distance between $p$ and the mbr-region of $e$. Figure 4 illustrates the computation of such a conservative approximation for a given index entry $e$ in a 2D feature space. First, we have to specify the maximum distance between the mbr-region of the index entry $e$ and any point in the vector space. It suffices to find for each point $p$ in the vector space the point $o \in \operatorname{subtree}(e)$ which is within the mbr-region of $e$ having the maximum distance to $p$. This can be done by considering partitions of the vector space which are generated as follows: in each dimension the space is split paraxially at the center of the mbr-region. As illustrated for the 2D example in Figure 4, we obtain partitions denoted by $N W, N E, S E$ and $S W$. In each of these partitions $P$, the vertex point of the mbr-region which lies within the diagonal-opposite partition is the mbr-region point which has the maximum distance to all points in $P$. In our example, for any point $p$ in $S W$ the maximum distance of $p$ to $e$ is the distance between $p$ and point $o_{1}$ in partition $N E$. Consequently, the hyperplane $\perp\left(q, o_{1}\right)$ is a conservative approximation of all hyperplanes between points within the mbr-region of $e$ and the points within the partition $S W$. In our example, the hyperplane associated with $e$ is composed by the three hyperplanes $\perp\left(q, o_{2}\right), \perp\left(q, o_{1}\right)$ and $\perp\left(q, o_{3}\right)$.

\subsection{Efficient Spatial Partial Pruning}

The basic idea of the partial pruning as introduced in 3.1 is to find for an intermediate index entry $e$ the point in $e$ with the lowest ranking count w.r.t. the hyperplanes that intersect $e$. An example of this situation is given in Figure 6 where $e$ is intersected by three hyperplanes $\perp\left(q, e_{1}\right), \perp\left(q, e_{n 2}\right)$ and $\perp\left(q, e_{3}\right)$ and $e_{1}$ corresponds to an intermediate index entry containing five points, whereas $e_{2}$ and $e_{3}$ correspond to data points. $\perp\left(q, e_{1}\right), \perp\left(q, e_{n 2}\right)$ and $\perp\left(q, e_{3}\right)$ partition $e$ into segments. For any segment $s$, the ranking count of all points $p \in s$ is equal. Thus, the minimum number of hyperplanes any point in $e$ is covered by, is the minimal ranking count of all segments of $e$. Therefore, the minimal ranking count of $e$ is 1 in our example. Thus, the ranking key of $e$ is increased by one.

However, this computation requires to examine $O\left(2^{m}\right)$ segments, where $m$ is the number of hyperplanes intersecting $e$. In [7], a similar approach is used, that requires to examine an exponential number of pruning intersection areas.

Next, we propose an efficient approach for partial pruning that is based on the following observations: 

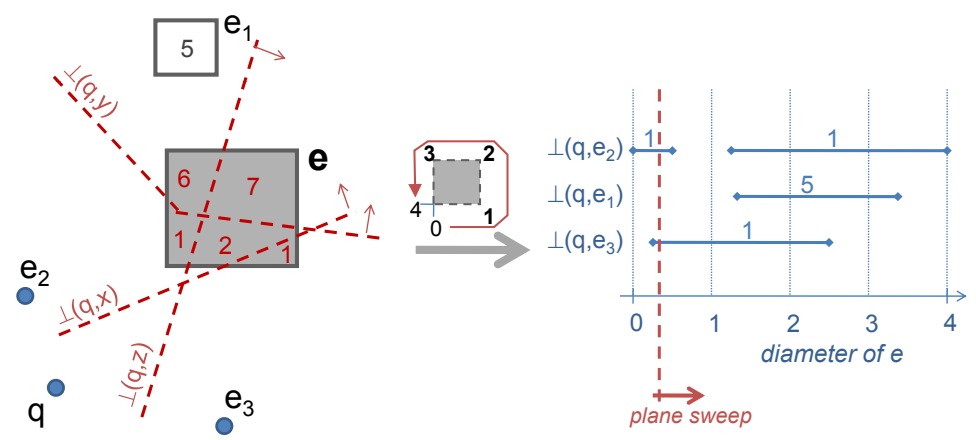

Fig. 6. Partial pruning based ranking count estimation strategy.

Lemma 1. If an intermediate index entry e contains the query point $q$, then the ranking count of $e$ is always 0 .

The above lemma is obvious, because if $e$ contains $q$, then $e$ may contain points that can be arbitrarily close to $q$, and thus, can have $q$ as their nearest neighbour regardless of the distance of $q$ to other points in the database.

Lemma 2. If an intermediate index entry e does not contain the query point $q$, then the minimal ranking key of all points of e is equal to the minimal ranking key of all points on the edges (i.e. the boundary) of $e$.

Proof. Assume an intermediate index entry $e$ and a query point $q$ outside of $e$. Let $p$ be an arbitrary point inside of $e$ and let $i$ denote the intersection of segment $[p, q]$ with the boundary of $e$. Assume that the ranking count of $i$ is $k$. Thus, by definition, at least $k$ points $p_{0}, \ldots, p_{k-1}$ are closer to $i$ than to $q$, i.e. $\operatorname{dist}(i, q)>\operatorname{dist}\left(i, p_{i}\right) \forall p_{i} \in$ $\left\{p_{0}, \ldots, p_{k-1}\right\}$. For each $p_{i} \in\left\{p_{0}, \ldots, p_{k-1}\right\}$, the following inequation holds:

$$
\operatorname{dist}(p, q)=\operatorname{dist}(p, i)+\operatorname{dist}(i, q)>\operatorname{dist}(p, i)+\operatorname{dist}\left(i, p_{i}\right)
$$

and the triangle inequality yields:

$$
\operatorname{dist}(p, i)+\operatorname{dist}\left(i, p_{i}\right)>\operatorname{dist}\left(p, p_{i}\right)
$$

Therefore, any point that is closer to $i$ than to $q$, is also closer to $p$ than to $q$. Thus the ranking count of $p$ is at least the ranking count of $i$. And since $i$ is located on the boundary of $e$, its ranking count is at least the ranking count of the minimum of the ranking counts of all points on the boundary of $e$.

For two dimensional data, we can use the above lemma to efficiently compute the partial pruning count of an index entry e using a plane sweep algorithm if $q$ is outside of the page region of $e$.

Since only the boundary of an intermediate index entry $e$ is required to determine its partial ranking count, we linearize $e$ to the interval $[0,4]$. Points on the lower edge 
of $e$ are represented by their relative position on that edge, points on the right edge of $e$ are presented by the relative position on that edge plus one, and so on (c.f. Figure 6). For each hyperplane approximation $\perp\left(q, e_{i}\right)$ of an index entry $e_{i}$ that intersects $e$, we determine all intersections of $e_{i}$ with $e^{2}$ and the respective regions of the boundary of $e$ that is pruned by $e_{i}$. In Figure 6 the boundary of $e$ is pruned by $e_{2}$ in the interval [2.3,3.4] and by $e_{1}$ in the interval [1.2,0.5], which is split into two intervals [1.2,4.0] and $[0.0,0.5]$. Now, the task is to find the minimum ranking count of all points on the boundary of $e$. The ranking count of a point $p$ on the boundary can be obtained by summing up all the ranking counts of all hyperplanes for which the corresponding interval covers $p$. Now the minimum ranking count of all points on the boundary can be computed using a plane sweep technique as depicted in Figure 6. During the sweep the global minimum of the ranking count is computed.

\subsection{Best-First Search Based Incremental RNN Ranking Algorithm}

In this section, we show how we explore the index such that the first results can be reported early without causing unnecessary page accesses. We start with an informal description of our solution before we present implementation details and pseudo code.

Similar to the TPL approach for RkNN queries our approach is based on a best-first search method exploiting a priority queue organizing the index entries to be explored. In contrast to the TPL approach, we propose to give the priorities to the index entries according to the estimated ranking count, i.e. entries with low ranking counts are ranked higher than entries with high ranking count. This means that entries containing objects with a low expected ranking position are explored before entries containing objects with a high expected ranking position. The rational for this strategy is that in this way we try to explore those entries first which contain potential candidates to be reported next from the ranking query.

For the organization of the index entries during the traversal of the index we maintain a priority queue $\mathcal{Q}$ storing entries with the corresponding estimated ranking count which are sorted in ascending order according to their estimated ranking count. Thereby we assume that the ranking count of each entry in this queue was generated by taking all current entries in the queue into account using the aforementioned strategies for increasing the ranking count.

The top element of the queue is the entry which has to be explored next. Whenever an entry $e$ is explored, i.e. $e$ is loaded from disk and is refined, we have to perform the following two steps: first, we have to update the ranking counts of all elements in the queue according to the children of $e$ and, second, the ranking counts of $e$ 's child elements have to be computed before we insert them into $\mathcal{Q}$.

For the first step, we have to determine those entries in $Q$ which could be affected by the refinement of $e$, i.e. for which the ranking count might be increased after refining $e$. Obviously, those entries which are completely behind the hyperplane representation of $e, \perp(q, e)$, must also be behind the hyperplane representations of each child of $e$ and, thus, their ranking count is not affected by the refinement of $e$. In the example

\footnotetext{
${ }^{2}$ Note that a hyperplane approximation can have at most four intersection with an mbr, due to its convex shape.
} 


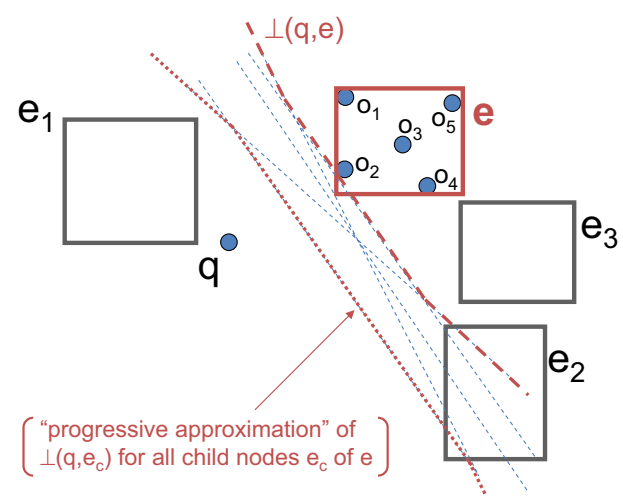

Fig. 7. Illustration of entries that are/are not affected by the refinement of an entry $e$.

shown in Figure 7, entry $e_{3}$ is not affected by the refinement of entry $e$ due to the above considerations. Furthermore, we can ignore those entries $e^{\prime}$ which cannot be behind a hyperplane of any object within subtree $(e)$, e.g. entry $e_{1}$ in the example in Figure 7, i.e. those entries $e^{\prime}$ for which the following statement holds:

$$
\exists p \in e^{\prime} . m b r: \operatorname{dist}(p, q)<\operatorname{MinDist}(p, e . m b r)
$$

Intuitively, those entries are not behind the "progressive approximation" of all hyperplanes $\perp\left(q, e_{c}\right)$ of child entries $e_{c}$ of entry $e$ (cf. Figure 7).

Entries which are affected by the refinement of $e$ are the remaining entries, i.e. those entries $e^{\prime}$ that fulfill both of the following two conditions:

$$
\exists p \in e^{\prime} . m b r: \operatorname{dist}(p, q) \leq \operatorname{MaxDist}(p, e . m b r)
$$

and

$$
\forall p \in e^{\prime} \cdot m b r: \operatorname{dist}(p, q) \geq \operatorname{MinDist}(p, e) .
$$

Each entry $e^{\prime}$ fulfilling the above two conditions, e.g. entry $e_{2}$ in our example in Figure 7, has to be checked against the hyperplane representation of each child of $e$. If the entry $e^{\prime}$ is behind the hyperplane representation of a child $e_{c}$ of $e$, its ranking counter will be increased by $\mid$ subtree $\left(e_{c}\right) \mid$.

For the second step, we have to determine the ranking counts of the children of $e$. For that purpose, we simply have to check all existing entries in $\mathcal{Q}$ and all other children of $e$ whether the current child $e_{c}$ of $e$ is behind the corresponding hyperplanes. If yes, the ranking count of $e_{c}$ is increased by the number of objects included in the subtree of the corresponding entry.

Finally, if the top entry $e$ in the queue $\mathcal{Q}$ is a point, i.e. $e \in \mathcal{D}$, the point can be output as a result only if $e$ is not beyond any progressive approximation of hyperplanes of child nodes of all $e^{\prime}$ that are currently in the queue, i.e. formally

$$
\forall e^{\prime} \in \mathcal{Q}: \operatorname{dist}(e, q)<\operatorname{MinDist}\left(e, e^{\prime} . m b r\right) .
$$


Otherwise, we need to refine any of those entries $e^{\prime} \in \mathcal{Q}$, for which this condition does not hold. As a consequence, $e$ might get a higher ranking count and might be shifted towards the end of $\mathcal{Q}$ or it may also maintain the top spot of $\mathcal{Q}$.

The pseudocode of the algorithm for the incremental RNN ranking is illustrated in Figure 8 providing the implementation details of the previously discussed steps. First, we initialize an empty result list "result" and the priority queue $\mathcal{Q}$ which stores index entries sorted in ascending order of their ranking count. Ties occuring in the priority queue are resolved by, first, prefering leaf index entries to directory index entries and, second, by sorting the entries in increasing distance to $q$.

The priority queue is initialized with the root of the index. For each call of the getNext method, we dequeue the first entry $e$ of $\mathcal{Q}$. If $e$ is a directory node, then it will be refined calling the refine routine depicted in Figure 9. During refinement, we first have to find all entries in $\mathcal{Q}$ that are candidates for having their ranking count increased due to the refinement of $e$ (see first step above). An entry $e^{\prime}$ is such a candidate, if there exists a point in the mbr of $e^{\prime}$ that is closer to $q$ than any point in $e$ (see the predicate in line 3 of the refinement procedure in Figure 9) and if $e^{\prime}$ has not already been reranked by $e$ (see the predicate in line 4 of the refinement procedure in Figure 9). These candidates are stored in a list updateI.

Additionally, we need all entries that are candidates for increasing the ranking count of one of the child entries of $e$ (see the second step above). An entry $e^{\prime \prime}$ is such a candidate, if it has not re-ranked e already (first comparison in line 6 of the refinement procedure in Figure 9) and its mbr contains a point that is closer to $q$ than a point in $e$ (second comparison in line 6 of the refinement procedure in Figure 9). These candidates are stored in a list updateII.

Lines 8-12 check for each child node $e_{c}$ of $e$ and element $e^{\prime} \in$ updateI, if $e$ re-ranks $e^{\prime}$ and increases the ranking count of $e^{\prime}$ if necessary. Analogously, lines 13-17 increase the ranking count of $e_{c}$, if an element $e^{\prime \prime} \in$ updateII re-ranks $e_{c}$.

Then, we increase the ranking count for each child entry $e_{c}^{\prime}$ of $e$ that is able to rerank $e_{c}$. Note that $e_{c}$ and $e_{c}^{\prime}$ may be identical, i.e. $e_{c}$ re-ranks itself. Finally $e_{c}$ is inserted into the queue $\mathcal{Q}$.

If the entry $e$ is a leaf entry, i.e. $e$ is an object, then $e$ obviously cannot be refined. However, we may not yet return $e$ as a result without further checking, because it may be re-ranked due to an entry that has not yet been refined. In that case, we need to scan the queue $\mathcal{Q}$ for an object that is a candidate for re-ranking $e$ by calling the refinementRound algorithm which is depicted in Figure 10 and refining (c.f. Figure 9) this object. If no such object exists, $e$ can be returned as the result of the current getNext()-call.

\section{Experimental Evaluation}

In this section, we present the results of our experiments. We start by explaining in detail the settings of our experiments and those of the competitors. Then, we show the results of our performance evaluation on multi-dimensional data. Finally, we evaluate the effect of the partial spatial pruning on 2D-datasets. 


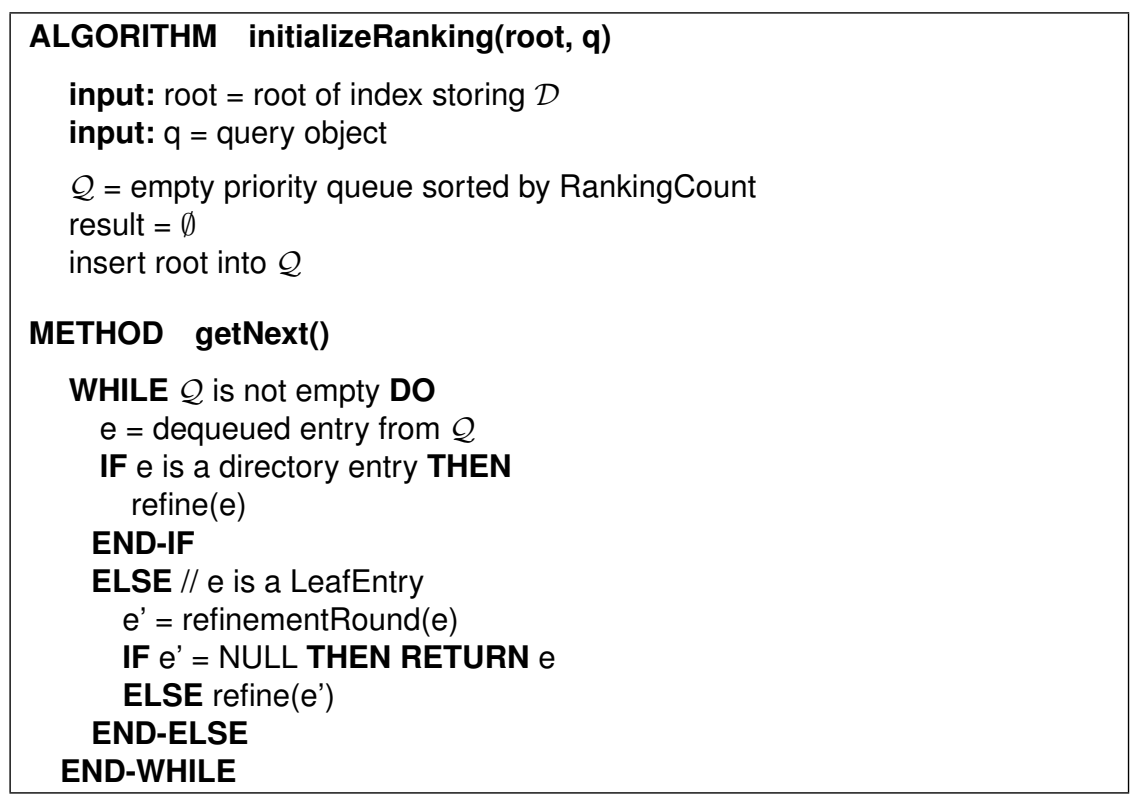

Fig. 8. Pseudocode of the incremental RNN ranking algorithm.

\subsection{Test Bed}

We compared our novel approach for computing an R $k \mathrm{NN}$ ranking, with two adaptions of the TPL [7] approach which is the current state-of-the-art algorithm for R $k \mathrm{NN}$ query processing. In fact, we applied two versions of the TPL approach for computing a ranking. The problem of the TPL approach is that we cannot predict the number of getNext()-calls beforehand. Thus, we do not know a suitable value of $k$ to answer all getNext()-calls.

The first variant, called TPL-Lazy, implements a lazy strategy assuming that we have a low number of getNext()-calls. It manages a result list which is initially empty and a counter $k_{c}$ which stores the current value of $k$ and is initialized with $k_{c}=1$. The entries in the result list are ordered by increasing ranking scores. For each call of the getNext() method, this variant checks the result list. If the result list is empty, TPL-Lazy computes a R $k \mathrm{NN}$ query with $k=k_{c}$ using the original TPL approach, adds the result of this query to the result list with a ranking score of $k_{c}$, and increments $k_{c}$. These three steps are processed iteratively until the result list is no longer empty. Last but not least, the TPL-Lazy method returns the next entry in the result list. Obviously, this variant only issues a new $\mathrm{R} k \mathrm{NN}$ query if necessary beginning with $k=1$ and successively incrementing the value of $k$. The costs for answering $l$ getNext()-calls are the sum of the costs of all queries for $k=1, \ldots$ necessary to answer the $l$ calls.

The second variant, called TPL-Eager, implements an eager policy assuming a higher but possible fixed maximum number of getNext()-calls. It simply assumes that the maximum number of getNext()-calls will be less than the number of result objects of a $\mathrm{R} k \mathrm{NN}$ query with a special value of $k_{\max }$, e.g. $k_{\max }=100$. Then, we only need 


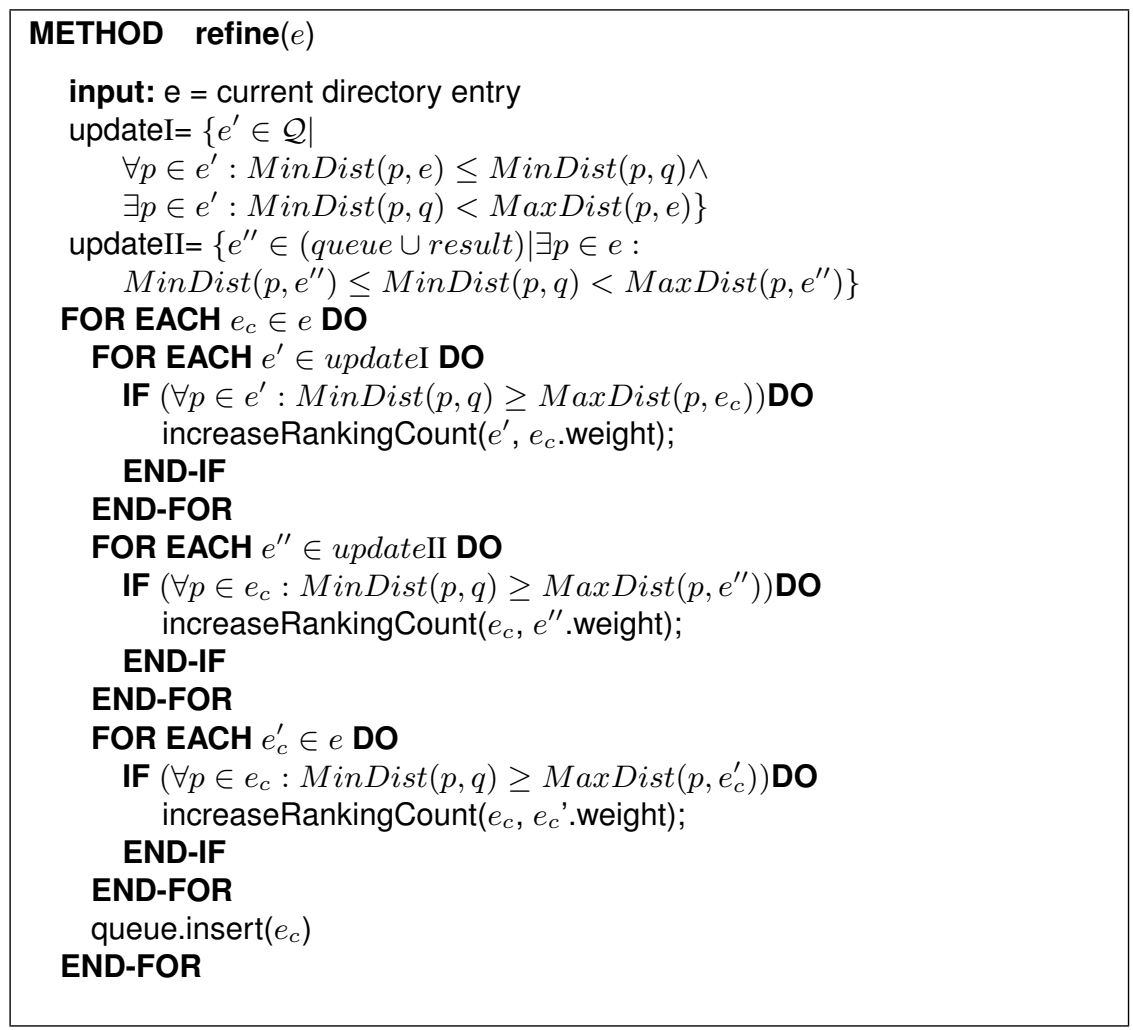

Fig. 9. Pseudocode of our refine algorithm.

to issue one $\mathrm{R} k_{\max } \mathrm{NN}$ query using the original TPL approach beforehand and sort the results according to their ranking score. Whenever a getNext()-call is issued (and as long as the assumptions stated above regarding the size of the result and the number of getNext()-calls hold), we can simply return the next object from the result list. The costs for answering $l$ getNext()-calls equal to the costs of answering the R $k_{\max } \mathrm{NN}$ query (again, as long as the result contains at least $l$ points). Let us note that there is no direct relationship between the number of $\operatorname{getNext()-calls~} l$ and the value $k_{\max }$. This makes it even harder for the TPL-Eager approach to guess a proper $k_{\max }$ value. In fact, to obtain a fair comparison, we computed the most optimistic scenario for the TPL-Eager variant: we first issued $l$ getNext()-calls with our new ranking method and obtained the ranking count of the resulting point of the last call. This count is the optimal $k_{\max }$ value for the TPL-Eager approach and we used this value in all our experiments. Thus, in realistic scenarios, the results of a TPL-Eager approach would be worse than presented here. All experiments are based on an $\mathrm{aR}^{*}$-Tree (aggregate version of $\mathrm{R}^{*}$-Tree) with a page size of $1 \mathrm{~K}$. Since all approaches are I/O bound we compared the number of disc pages accessed during the execution of 500 sample $\mathrm{R} k \mathrm{NN}$ queries and averaged the results. 


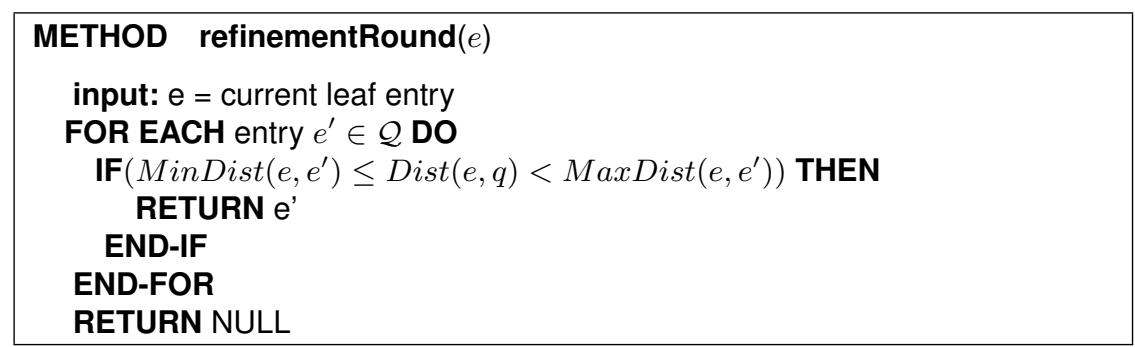

Fig. 10. Pseudocode of the refinement round.

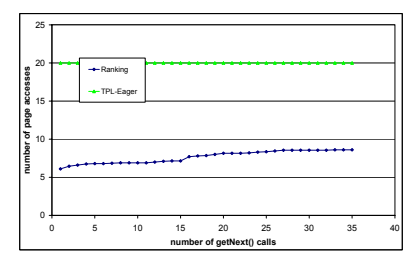

(a) Comparison to TPL-Eager.

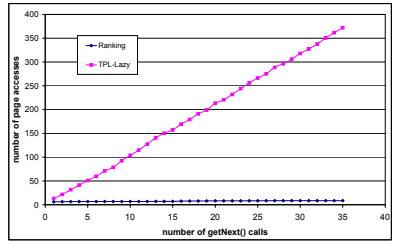

(b) Comparison to TPL-Lazy.

Fig. 11. Comparison of our $\mathrm{R} k \mathrm{NN}$ ranking with the competitors on uniformly distributed data.

\subsection{Performance Evaluation}

Synthetic Data We used two synthetic datasets to compare the performance of our ranking algorithm with the two variants of TPL. The first dataset contains 10,000 uniformly distributed 2D points. Figure 11 displays the performance of the competitors w.r.t. the number of getNext()-calls. As expected, the performance of the TPL-Eager approach (c.f. Figure 11(a))is constant as long as the number of getNext()-calls is smaller than the number of results of the $\mathrm{R} k_{\max } \mathrm{NN}$ query issued beforehand (which is the case in our scenario - see above). Nevertheless, our ranking algorithm clearly outperforms this TPL variant in terms of query execution times. In fact, the costs of our approach increase only slightly with successive getNext()-calls. In addition, it should be noted that TPL-Eager would need to issue a new R $k$ NN query with a considerably higher value of $k$ if we have more than 35 getNext()-calls because TPL-Eager was optimized for 35 results. Thus, in that case, we would have a jump for the TPL-Eager approach at the 36th getNext()-call while the costs of our ranking algorithm will most likely evolve like in the range of the first 35 getNext()-calls. On the other hand, the costs for the TPLLazy variant (cf. Figure 11(b)) increase much faster than the costs of our new ranking algorithm. Again our approach clearly outperforms the competitor in terms of query execution times. Note that the performance of our ranking algorithm is of course the same in both Figures 11(a) and 11(b).

A similar observation can be obtained from Figure 12 which displays the performance of the competitors on a 2D synthetic dataset that contains 10,000 points clustered into four different clusters. The only obvious difference is that here, the TPLEager approach performs much better than on the uniform dataset. As illustrated in Figure 14(a), our ranking algorithm outperforms the TPL-Eager variant only for the 


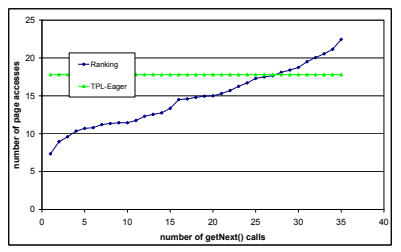

(a) Comparison to TPL-Eager.

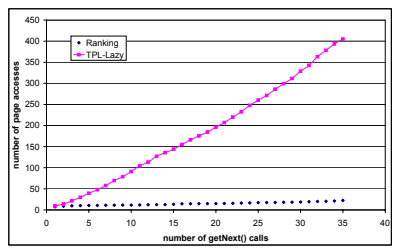

(b) Comparison to TPL-Lazy.

Fig. 12. Comparison of our R $k \mathrm{NN}$ ranking with the competitors on clustered data.

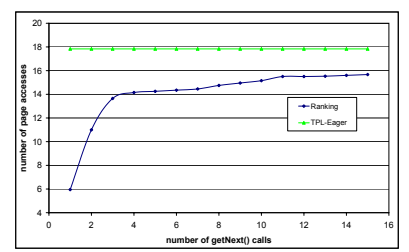

(a) Comparison to TPL-Eager.

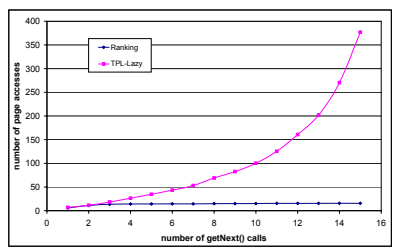

(b) Comparison to TPL-Lazy.

Fig. 13. Comparison of our $\mathrm{R} k \mathrm{NN}$ ranking and the competitors on $5 \mathrm{D}$ gene expression data.

first 27 getNext()-calls. In this setting, the TPL-Eager slightly outperforms our ranking algorithm for 30 to 35 getNext()-calls. However, please note that, first, the TPL-Eager approach was implemented with the most optimistic assumptions and can be expected to perform considerably worse in a more realistic scenario where the perfect $k_{\max }$ value can usually not be determined beforehand. Second, as explained above, TPL-Eager was optimized for 35 results. If we had more than 35 getNext()-calls, then TPL-Eager would be required again to compute a new $\mathrm{R} k \mathrm{NN}$ query with a considerably higher value of $k$ which would cause significantly higher costs from the 36th getNext()-call on until the next jump limit is reached.

On the other hand, in comparison to the TPL-Lazy variant (cf. Figure 14(b)) our ranking algorithm again performs much better and significantly outperforms the competitor in terms of query execution times. Again, the performance of our ranking algorithm is of course the same in both Figures 14(a) and 14(b).

Real-world Data We also tested our novel ranking algorithm on real-world data. In Figure 13 the performance of our ranking algorithm is compared with the performances of the TPL-Eager variant (cf. 13(a)) and the TPL-Lazy variant (cf. 13(b)) on a dataset that features the expression level of approx. 6,000 genes under 5 conditions. The result on this 5D dataset is similar to the results on the synthetic datasets reported above. Again, our ranking algorithm clearly outperforms the TPL-Lazy approach. Analogously, the difference to the TPL-Eager approach is less significant but still considerable. It should again be noted that the TPL-Eager variant assumes the most optimistic scenario for its application which is most likely not a realistic setting, and, thus, it can be expected that TPL-Eager performs less accurate in most applications. 


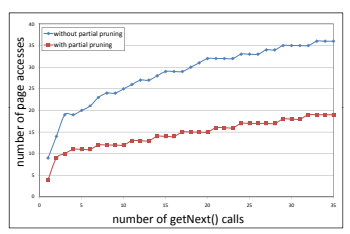

(a) Uniformly data.

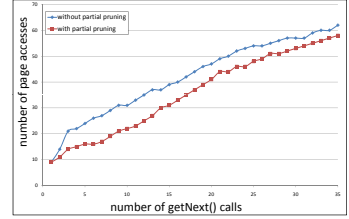

(b) Clustered data.

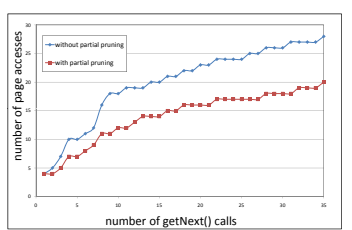

(c) Real data.

Fig. 14. Effect of the spatial partial pruning.

\subsection{Effect of the Spatial Partial Pruning}

We evaluated our spatial partial pruning technique using a uniformly and a clustered 2D-dataset each containing 10,000 datapoints. The results are depicted in Figure 14. Notice that the number of page accesses is reduced by using partial pruning in both experiments. The effect however, is much more significant on the uniformly distributed dataset. Finally, we performed the same experiment on a real world dataset extracted from the Forest Cover Type dataset, retrieved from the UCI KDD repository [15] consisting of 10,000 2D-points. The result in Figure 14(c) shows that the spatial partial pruning technique reduces the number of page accesses by about $25 \%$.

\subsection{Summary}

To summarize the results of our experimental evaluation, our novel ranking algorithm outperforms both adaptions of the existing TPL algorithm to the ranking problem, TPLEager and TPL-Lazy, significantly in terms of query execution times. While TPL-Eager seems to be competitive (if at all) only for a higher number of getNext()-calls, TPL-Lazy seems to be competitive (if at all) only for a very low number of getNext()-calls. This result is quite intuitive because TPL-Eager tries to estimate the worst-case by precomputing the maximum number of required results for a maximum number of getNext()calls by computing one $\mathrm{R} k_{\max } \mathrm{NN}$ query. Thus, the more the number of getNext()-calls reaches the number of resulting objects of the $\mathrm{R} k_{\max } \mathrm{NN}$ query, the more the costs for the $\mathrm{R} k_{\max } \mathrm{NN}$ query pay off. Otherwise, TPL-Eager caused a large portion of unnecessary costs to compute a large number of results that are not needed. On the other hand, TPL-Lazy assumes the best case of very few getNext()-calls and, thus, computes results only if necessary by consecutively issuing a $\mathrm{R} k \mathrm{NN}$ query with increasing $k$. Obviously, as long as the number of consecutive $\mathrm{R} k \mathrm{NN}$ queries, with increasing $k$ necessary to report results, is small, i.e. the number of getNext()-calls is low, this strategy pays off. Otherwise, TPL-Lazy constantly recomputes $\mathrm{R} k \mathrm{NN}$ queries with the next higher value for $k$ which produces a lot of redundant results w.r.t. the previously computed queries.

Our ranking algorithm obviously performs best because it does not assume worst- or best-cases but focuses on computing the ranking incrementally. Since in a ranking query scenario, it is not known beforehand, how often the method getNext() is called, this is the most efficient solution in the general case but also - as our experiments illustrate in the borderline cases where either TPL-Eager or TPL-Lazy perform best. 


\section{Conclusions}

In this paper, we formalize a novel ranking problem, the reverse nearest neighbor (RNN) ranking and propose an original solution for it. Our solution extends existing methods for RNN query processing in the following important aspects. First, the mutual-pruning strategy of existing approaches is generalized and adapted so that it can be applied already on higher levels of the index and it can be applied to estimate the ranks of an index entry, rather than just for pruning. Second, we incorporated the idea of self-pruning and explored how this concept can be applied to estimate the ranking of index entries. Third, we explored the concept of partial pruning and derived an efficient solution to integrate the estimation of ranking counts based on this concept for 2D spatial data. Last but not least, we proposed an incremental algorithm for the RNN ranking problem that is based on both introduced ranking estimations. Our experimental evaluation confirms that our new solution outperforms existing methods adapted for the new problem significantly in terms of query execution times.

\section{References}

1. Lazaridis, I., Mehrotra, S.: Progressive approximate aggregate queries with a multiresolution tree structure. In: Proc. SIGMOD. (2001)

2. Papadias, D., Kalnis, P., Zhang, J., Tao, Y.: Efficient olap operations in spatial data warehouses. In: Proc. SSTD. (2001)

3. Korn, F., Muthukrishnan, S.: Influenced sets based on reverse nearest neighbor queries. In: Proc. SIGMOD. (2000)

4. Yang, C., Lin, K.I.: An index structure for efficient reverse nearest neighbor queries. In: Proc. ICDE. (2001)

5. Stanoi, I., Agrawal, D., Abbadi, A.E.: Reverse nearest neighbor queries for dynamic databases. In: Proc. DMKD. (2000)

6. Singh, A., Ferhatosmanoglu, H., Tosun, A.S.: High dimensional reverse nearest neighbor queries. In: Proc. CIKM. (2003)

7. Tao, Y., Papadias, D., Lian, X.: Reverse kNN search in arbitrary dimensionality. In: Proc. VLDB. (2004)

8. Lee, K.C.K., Zheng, B., Lee, W.C.: Ranked reverse nearest neighbor search. IEEE TKDE 20(7) (2008) 894-910

9. Achtert, E., Böhm, C., Kröger, P., Kunath, P., Pryakhin, A., Renz, M.: Efficient reverse k-nearest neighbor search in arbitrary metric spaces. In: Proc. SIGMOD. (2006)

10. Achtert, E., Böhm, C., Kröger, P., Kunath, P., Pryakhin, A., Renz, M.: Approximate reverse k-nearest neighbor search in general metric spaces. In: Proc. CIKM. (2006)

11. Tao, Y., Yiu, M.L., Mamoulis, N.: Reverse nearest neighbor search in metric spaces. IEEE TKDE 18(9) (2006) 1239-1252

12. Guttman, A.: R-Trees: A dynamic index structure for spatial searching. In: Proc. SIGMOD. (1984) 47-57

13. Beckmann, N., Kriegel, H.P., Schneider, R., Seeger, B.: The R*-Tree: An efficient and robust access method for points and rectangles. In: Proc. SIGMOD. (1990) 322-331

14. Berchtold, S., Keim, D.A., Kriegel, H.P.: The X-Tree: An index structure for highdimensional data. In: Proc. VLDB. (1996)

15. Hettich, S., Bay, S.D.: The uci kdd archive. (1999) 